\title{
GROWTH OF InN BY MBE
}

\author{
W.-L. Chen*, R. L. Gunshor*, Jung Han**, K. Higashimine***, and N. Otsuka*** \\ *School of Electrical and Computer Engineering, Purdue University, \\ West Lafayette, IN 47907 \\ **Sandia National Laboratories, Albuquerque, NM 87185 \\ ***Japan Advanced Institute of Science and Technology, Ishikawa, Japan
}

\begin{abstract}
A series of experiments were performed to explore the growth of $\mathrm{InN}$ by Molecular Beam Epitaxy (MBE). The growth conditions were optimized based on the study of RHEED during growth and InN dissociation experiments. Characterization of the $\mathrm{InN}$ thin films were performed by various techniques such as TEM and XRD.
\end{abstract}

\section{Introduction}

InN containing compounds such as the GaInN ternary are of importance for the fabrication of light emitting devices [1-3]. However, phase separation is known to prevent the homogeneous incorporation of high In-content GaInN for longer wavelength applications [4-5]. The behavior of pure InN growth might help to shed light on the mechanism of the formation of high concentration of InN in the ternary. $\mathrm{InN}$ has been prepared by various techniques [6-13]. However, $\mathrm{InN}$ normally shows poor optical properties, a high background carrier concentration and poor crystalline properties with indium clusters found from the x-ray diffraction spectrum.

For the MBE technique, the most important growth parameters are the flux levels and growth temperature. In the study reported here, RHEED behavior during growth and $\mathrm{InN}$ thermal dissociation experiments following growth have been performed to optimize the growth parameters. The results of the characterization of InN films by various means are presented in this report.

\section{Experiment}

A Perkin-Elmer 430 MBE system was used for the growth of InN. In flux was measured by a crystal monitor located at the growth sample position. An RF nitrogen plasma cell (Oxford CARS-25) was used to supply the active nitrogen. The substrates used were 2-2.5 $\mu \mathrm{m}$ thick GaN epilayers grown on c-plane sapphire by MOCVD at Sandia National Labs and, a $1 \mu \mathrm{m}$ thick molybdenum layer was evaporated on the backside of the substrates to absorb the radiation from the filament. Substrates were screw mounted on an unbonded molybdenum block. Immediately before being introduced into the growth chamber, the substrates were preheated in a preparation chamber to $350^{\circ} \mathrm{C}$ for 10 minutes to reduce outgassing from the block when it was heated in the growth chamber. 
The growth temperature was measured by a thermocouple on the back of the substrate and was calibrated by the eutectic point of gold-germanium at $356^{\circ} \mathrm{C}$. The plasma cell was in general operated at $500 \mathrm{~W}$ with a nitrogen flow rate of $0.8-1.2 \mathrm{sccm}$. The chamber pressure was in the $2.5-5 \times 10^{-5}$ Torr range during growth. The nominal active nitrogen flux under this condition was measured by GaN RHEED intensity oscillations under $\mathrm{N}$-limited growth conditions. The $\mathrm{GaN}$ growth rate was limited by the nitrogen flux at substrate temperatures lower than $750^{\circ} \mathrm{C}$, above which dissociation became a factor. The available active nitrogen flux was determined to be $3.7 \times 10^{14}$ atoms $/ \mathrm{cm}^{2} / \mathrm{sec}$ which was equivalent to $0.36 \mathrm{ml} / \mathrm{sec}$ for $\mathrm{InN}$ if all the active nitrogen were incorporated. In the experiments, the In flux ranged from 0.02 to $0.3 \mathrm{ml} / \mathrm{sec}$.

During the growth, RHEED provided real time observation of the growth front. Depending on the fluxes and substrate temperature, InN showed three distinct RHEED patterns during growth. Fig.1 illustrates three different RHEED patterns in the <11$20>$ direction. In general higher N/In flux ratio and lower temperature results in pure $3 \mathrm{D}$ bright spots. At a lower N/In flux ratio and higher temperature, the RHEED pattern is a streaky 2-fold pattern. For conditions in between, the RHEED pattern shows both streaky 2 -fold streaks and bright $3 \mathrm{D}$ spots at the same time.
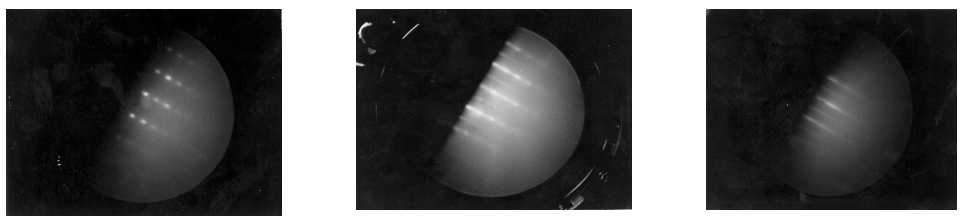

Fig.1 Three RHEED patterns during the growth of InN: 1-fold spots, 2-fold + spots, and streaky 2-fold.

As soon as InN had been nucleated on the GaN surface, the RHEED changed from the GaN streaky pattern to 3D spots. After that, the RHEED continued with the 3D pattern or gradually changed to one of the two different RHEED patterns depending on the growth conditions. The "better" growth region judging from the optical absorption and X-ray Diffraction (XRD) characterization was within the pure 3D spots region.

All the better quality InN films, as judged by XRD and absorption characteristics, were grown with 3D RHEED patterns exhibited throughout the whole growth course. We find that the low N/In ratio and/or high growth temperature associated with streaky RHEED are ultimately detrimental to film quality. It is interesting to note that when In was deposited on InN at $350^{\circ} \mathrm{C}$ (our normal growth temperature) the RHEED observed was also streaky. We could not distinguish the streak spacing of $\mathrm{InN}$ from that of pure In. Therefore we speculated that the streaky 2 fold RHEED during the InN growth could also be associated with the presence of pure In at the growth surface. For temperatures above $430^{\circ} \mathrm{C}$ where $\mathrm{InN}$ dissociation becomes significant, an In rich surface results even for higher $\mathrm{N} / \mathrm{In}$ ratios.

In the past the thermal dissociation of InN has been studied under high pressure and vacuum [14-16], with some disagreement between the reported results. The possible reason for the discrepancy could be the variations in crystal quality. Since the high nitrogen partial pressure over $\mathrm{InN}$ is one of the major reasons why $\mathrm{InN}$ is extremely 
difficult to grow, we performed a series of dissociation experiments in the MBE growth chamber.

The Quadrapole Mass Analyzer (QMA) has been previously used for the study of nitride growth in an MBE system previously [17]. In a dissociation experiment, an InN thin film (typically $0.3 \mathrm{um}$ ) was first grown on the GaN epilayer as described above. The sample was then gradually heated at a constant rate. The signals at atomic mass 14, 28, and 115 and substrate temperature were recorded. At the same time, RHEED pictures were taken. Fig.2 illustrates the QMA signal intensities vs. temperature at different stages of the experiment. There are several interesting features in the plots:

Indium (115) and nitrogen signals (14 and 28) started to rise at about $430^{\circ} \mathrm{C}$, as did chamber pressure. InN was previously reported to dissociate rapidly at $500^{\circ} \mathrm{C}$ [14]. In this experiment, as the pressure shows in Fig.2, there is considerable dissociation at $450^{\circ} \mathrm{C}$. The nitrogen signals reached a local maximum at $570^{\circ} \mathrm{C}$ and then started to drop. However the In intensity kept increasing as the temperature rose. This drop in nitrogen likely represents the elimination of the InN crystal. The nitrogen escaping from the substrate started to drop after this point. However, In did not escape from the film surface immediately after the $\mathrm{InN}$ decomposed. The rapid increase of the nitrogen signals above $750^{\circ} \mathrm{C}$ can be attributed to the decomposition of $\mathrm{GaN}$ as well as the outgassing of the growth manipulator.

The RHEED evolution during the dissociation experiments provided further evidence that when InN dissociated, the In tended to stay on the surface. When the InN started to dissociate at $430^{\circ} \mathrm{C}$, the RHEED remained as 3D spots with the intensity dropping as the temperature rose. The intensity kept dropping until the local maximum of nitrogen signals was reached at $570^{\circ} \mathrm{C}$. At the same time, while the RHEED intensity dropped, the streaky pattern started to develop. The appearance of streaks is consistent with the idea that at this point most InN had decomposed and an accumulation of In on the surface lowered the RHEED intensity. It should be noted that when the RHEED of InN was studied during growth, we observed that the RHEED intensity of streaky patterns tended to decrease faster than did the 3D or mixed patterns. As a result, we attributed the decrease to the accumulation of In.

At a temperature $450^{\circ} \mathrm{C}$, the loss of In due to dissociation was compatible to the In flux typically employed during growth. It should be recalled that the In flux during growth is limited by the available active nitrogen. This implied that the growth should be conducted at temperatures much lower than $450^{\circ} \mathrm{C}$ to avoid the decomposition of InN.

\section{Results}

For films grown with a spotty RHEED pattern, AFM studies showed grainy features which are consistent with the island growth mode. The diameter of the grains ranges from 0.2 to $0.3 \mu \mathrm{m}$. AFM was also used to resolve the morphology of a sample exhibiting the mixed RHEED patterns (streaks and spots) described previously. The AFM picture shows that the surface is essentially a flat plane with a few islands of different height scattered over the surface. The RHEED streaks are interpreted as caused by the flat plane, while the spots were from the transmission type diffraction when electrons passed through the isolated islands. 
Optical transmission experiments were conducted to evaluate the InN optical quality. Fig. 3 shows the transmitted light intensity in the vicinity of bandgap of a reddish film. (We found that the film color was useful in judging the relative quality of InN samples.) A relatively broad band edge was seen; the absorption characteristic is consistent with the red appearance of the film. A considerable absorption below the bandgap was also observed. Depending on the growth conditions, InN showed a red color with different degrees of transparence. Films grown at temperatures around $350^{\circ} \mathrm{C}$ with low In flux showed a brighter red color. Films grown under higher temperatures or lower N/In flux ratios tended to show a darker color tending to black when the below bandgap absorption is strong. The films with brighter red color also exhibited narrower XRD peak widths and improved microstructures as illustrated by the TEM images.

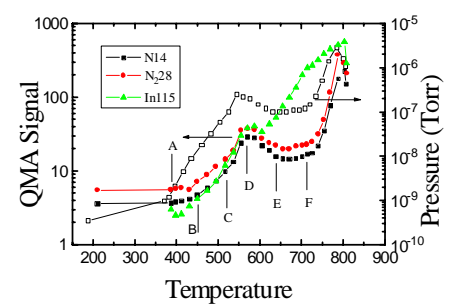

Fig.2 Dissociated $\mathrm{In}$ and $\mathrm{N}$ as a function of temperature.
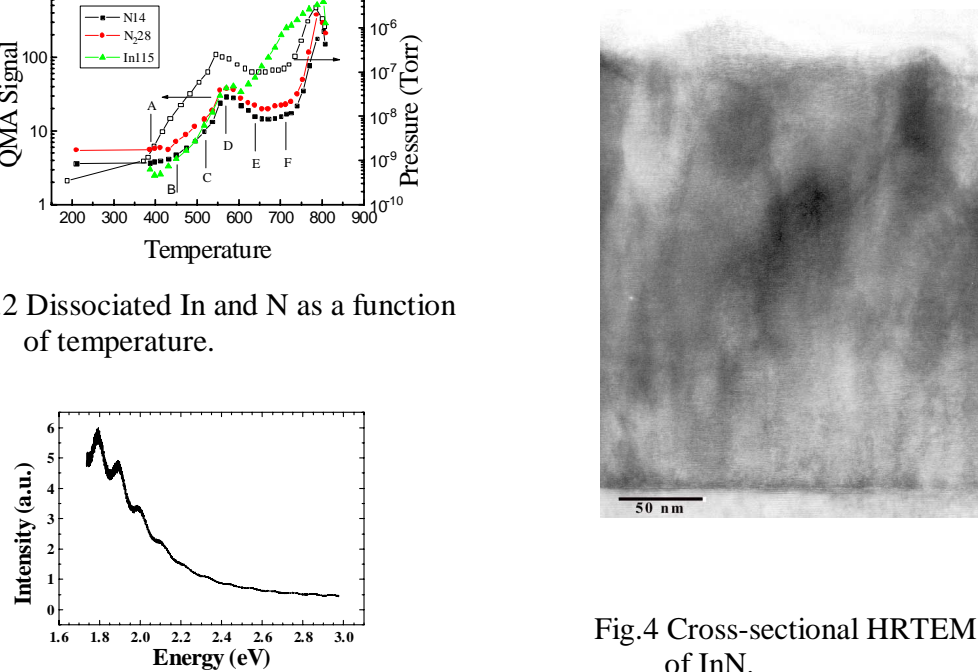

Fig.3 Optical absorption of a typical reddish $\mathrm{InN}$.

Fig. 4 Cross-sectional HRTEM of InN.

Table1 List of InN growth conditions and results.

\begin{tabular}{|l|l|l|l|l|l|}
\hline Sample & $\begin{array}{l}\text { In flux } \\
(\mathrm{ml} / \mathrm{sec})\end{array}$ & $\begin{array}{l}\text { Temp } \\
\left({ }^{\circ} \mathrm{C}\right)\end{array}$ & $\begin{array}{l}\text { XRD } \\
\text { FWHM }\end{array}$ & $\begin{array}{l}\text { Growth } \\
\text { interruption }\end{array}$ & $\begin{array}{l}\text { Morphology/ } \\
\text { Color }\end{array}$ \\
\hline InN071398 & 0.05 & 355 & 1705 & 60 secs per 12 ml & In balls, red \\
\hline InN-8 & 0.05 & 330 & 1691 & 90secs per 12 ml & Wavy, red \\
\hline InN-9 & 0.05 & 380 & 1613 & 90 secs per 12 ml & dark \\
\hline InN-10 & 0.05 & 405 & 1397 & 90 secs per 12 ml & dark \\
\hline InN-11 & 0.03 & 350 & 1314 & No & dark \\
\hline InN-12 & 0.03 & 350 & 1631 & 60 secs per 12 ml & Red \\
\hline InN-13 & 0.08 & 350 & 2539 & 60 secs per 12 ml & dark \\
\hline InN-14 & 0.02 & 350 & 1102 & 60 secs per 12 ml & Red \\
\hline
\end{tabular}


The crystalline quality was analyzed by XRD and TEM. Table1 lists a group of films grown. The (0002) rocking curve diffraction was performed on a double crystal $\mathrm{X}$-ray diffraction system. All films are $0.2-0.3 \mu \mathrm{m}$ thick. Fig.4 is a High Resolution Transmission Electron Microscope (HRTEM) image taken from a cross-sectional sample of the InN layer grown under the optimized condition. The image shows that lattice fringes of basal planes of the InN crystal are seen continuously in the direction parallel to the growth plane from the bottom to the top of the layer, though these basal plane images are somewhat wavy. In the case of other InN layers whose growth conditions were not optimized, HRTEM images show distinct columnar-like structures, and lattice fringes of basal planes are discontinuous between neighboring columns with void-like images in boundary regions between columns. These HRTEM observations suggest that the optimization of the growth condition has improved the alignment of orientations of InN nuclei and lead to nearly single crystalline epitaxial layers as the growth has proceeded.

\section{Discussion}

Films grown at temperatures higher than $430^{\circ} \mathrm{C}$ are all black in appearance although the XRD width could be compatible to those with red color. For these films In droplets are usually found on the surface. The accumulation of In can be partly explained by the dissociation of $\mathrm{InN}$ at temperatures above $430^{\circ} \mathrm{C}$ as illustrated in Fig.2.

It was found that a high $\mathrm{N} / \mathrm{In}$ flux ratio is required in order to obtain a reddish $\mathrm{InN}$ film. For a growth temperature of $355^{\circ} \mathrm{C}$, In balls are seen on the surface although the $\mathrm{N} / \mathrm{In}$ ratio is seven. To avoid the formation of In droplets, N/In needs to be increased above seven; the observation suggests that it is more energetically favorable for indium to form indium clusters rather than the InN compound.

A periodic growth interruption seems to effectively improve the film color. For every 12 monolayer deposition, the In shutter is closed for 60 or 90 seconds while the nitrogen flux is on. As seen in Table1, we find that the interruption appears more effective than reducing the In flux without periodic growth interruption.

Thermodynamic theory has been applied to build models for the growth of IIInitrides [18]. In the cited equilibrium theory, the formation of indium droplets is determined by comparing the equilibrium indium partial pressure to the partial pressure given by the vapor pressure of In over liquid In at the growth temperature. The approach implies the assumption that the formation of $\mathrm{InN}$ has priority over the formation of In droplets. The results in [18] showed that In condensation does not occur at regions where the N/In flux ratio is larger than one. However, our experimental data indicates that indium droplets can still be formed even when the $\mathrm{N} /$ In flux ratio is much greater than one. Therefore the higher nitrogen flux does not guarantee the lack of indium condensation. Kinetic processes such as the surface mobility of the adatoms or barriers for the formation of compound could be possible factors which affect the InN MBE growth. In general, growth temperature plays an important role in these complex kinetic processes. Higher growth temperature is preferred to overcome such energy barriers if present. However, higher decomposition rates also accompany higher temperatures, calling for more active nitrogen to compensate. Unfortunately it is very difficult to get abundant active nitrogen in a $\mathrm{MBE}$ chamber; the highest temperature is limited to values under $400^{\circ} \mathrm{C}$ in our system. 


\section{Conclusion}

InN thin films have been grown on GaN/sapphire epilayers. The growth conditions are optimized based on a RHEED study and InN dissociation experiments. Films have been characterized by various techniques. The results indicate that seeking a stronger active nitrogen source is important to increase growth rate and growth temperature. Another direction to improve the film quality could possibly be to seek a possible surfacant for InN growth.

\section{Acknowledgement}

The authors acknowledge the technical support of Dr. A. Waag, Prof. A. Nurmikko, H. Zhou, Y.K. Song and Prof. A.K. Ramdas, M. Seong, Dr. J. Tsao and D. Lubelski. W.L. Chen and R.L. Gunshor are grateful for financial support from the Purdue Research Fundation.

\section{References}

[1] S. Strite, H. Morkoç, J. Vac. Sci. Technol. B 10, 1237 (1992).

[2] S. Nakamura, M. Senoh, N. Iwasa, S. Nagahama, T. Yamada, and T. Mukai, Jpn. J. Appl. Phys., 34, L1332 (1995).

[3] S. Nakamura, M. Senoh, S. Nagahata, N. Iwasa, T. Yamada, T. Matsushita, H. Kiyoku, Y. Sugimoto, Jpn. J. Appl. Phys. 35, L74 (1996).

[4] M. Shimizu, K. Hiramatsu, and N. Sawaki, J. Cryst. Growth 145, 209 (1994).

[5] R. Singh, D Doppalapudi, T.D. Moustakas, and L.T. Romano, Appl. Phys. Lett. 70, 089 (1997).

[6] K. Kubota, Y. Kobayashi, and K. Fujimoto, J. Appl. Phys. 66, 2984 (1989).

[7] C.R. Abernathy, S.J. Pearton, F. Ren, and P.W. Wisk, J. Vac. Sci. Technol. B11, 179 (1993).

[8] T.J. Kistenmacher, S.A. Ecelberger, and W.A. Bryden, J. Appl. Phys. 74, 1684 (1993).

[9] Y. Sato and S. Sato, J. Cryst. Growth 144, 15 (1994).

[10] Q. Guo, T. Yamamura, A. Yoshida, and N. Itoh, J. Appl. Phys. 75, 4927 (1994).

[11] W.E. Hoke, P.J. Lemonias, and D. G. Weir, J. Cryst. Growth 111, 1024 (1991).

[12] Y. Pan, W. Lee, C. Shu, H. Lin, C. Chiang, H. Chang, D. Lin, M. Lee, W. Chen, Jpn. J. Appl. Phys. 38, 645 (1999).

[13] S. Yamaguchi, M. Kariya, S. Nitta, T. Takeuchi, C. Wetzel, H. Amano, and I. Akasaki, J. Appl. Phys. 85, 7682 (1999).

[14] J.W. Trainor and K. Rose, J. Electron. Mater. 3, 821 (1974).

[15] A.M. Vorb'ev, G.V. Evseeva, and L.V. Zenkevich, Russ. J. Phys. Chem. 45, 1501 (1971).

[16] R.D. Jones and K. Rose, J. Phys. Chem. Solids 48, 587 (1987).

[17] R. Held, D.E. Crawford, A.M. Johnston, A.M. Dabiran, and P.I. Cohen, J. Electron. Mater. 26, 272 (1997).

[18] A. Koukitu and H. Seki, Jpn. J. Appl. Phys. 36, L750 (1997). 